Buzalaf, M.A.R. 30

Carvalho, T.S. 18

Charone, S. 30

Cheng, L. 38

Clarkson, B.H. 11

Crielaard, W. 1

Devine, D.A. 46

Exterkate, R.A.M. 1, 11

He, L. 38
Head, D.A. 46

Li, J. 38

Lussi, A. 18

Marsh, P.D. 46

ten Cate, J.M. 3, 55

Tjäderhane, L. 30

van Loveren, C. 1

Zaura, E. 55

Zhou, X. 38

\title{
Subject Index Vol. 49, Suppl. 1, 2015
}

Allostasis 55

Antimicrobial agents 46

Arginine 18

Biofilm 46

Caries 3, 30

Casein 18

Cavitated lesions 11

Chitosan 18

Chlorhexidine 30

Cross-linking 30

Cysteine cathepsin 30

Dental caries 38

Ecosystem 55

Erosion 30

Erosive tooth wear 18

Fluoride 18

Inhibition 30

Matrix metalloproteinase 30
Microbiome 3

Modelling 46

Mucin 18

Natural products 38

Oral health 55

- microbiome 46

pH cycling 3

Plaque control 46

Polyphenol compounds 38

Precavitated lesions 11

Prevention 3, 18

Remineralization 11

Salivary pellicle 18

Sodium hexametaphosphate 18

Stannous fluoride 18

Titanium tetrafluoride 18

Toothpastes 3

Traditional herbs 38 